\title{
Feature Generation and Dimensionality Reduction using the Discrete Spectrum of the Schrödinger Operator for Epileptic Spikes Detection
}

\author{
Abderrazak Chahid ${ }^{1}$,Turky Nayef Alotaiby $^{2}$, Saleh Alshebeili ${ }^{3}$, and Taous-Meriem Laleg-Kirati ${ }^{1, \star}$ \\ ${ }^{1}$ Computer Electrical and Mathematical Science and Engineering (CEMSE) division, \\ King Abdullah University of Science and Technology (KAUST), KSA. \\ ${ }^{2}$ King Abdulaziz City for Science and Technology (KACST), Riyadh, KSA. \\ ${ }^{3}$ King Saud University (KSU), Riyadh, KSA. \\ ^ Corresponding author: taousmeriem.laleg@kaust.edu.sa
}

\begin{abstract}
Epilepsy is a neurological disorder classified as the second most serious neurological disease known to humanity, after stroke. Magnetoencephalography (MEG) is performed to localize the epileptogenic zone in the brain. However, the detection of epileptic spikes requires the visual assessment of long MEG recordings. This task is time-consuming and might lead to wrong decisions. Therefore, the introduction of effective machine learning algorithms for the quick and accurate epileptic spikes detection from MEG recordings would improve the clinical diagnosis of the disease. The efficiency of machine learning based algorithms requires a good characterization of the signal by extracting pertinent and discriminative features. In this paper, we propose new sets of features for MEG signals. These features are based on a Semi-Classical Signal Analysis (SCSA) method, which allows a good characterization of peak shaped signals. Moreover, this method improves the spike detection accuracy and reduces the feature vector size. We could achieve up to $92.52 \%$ and $\mathbf{8 9 . 1 0 \%}$ in average sensitivity and specificity, respectively. We use the 5-folds cross-validation applied to a balanced dataset of 3104 frames, extracted from eight healthy and eight epileptic subjects with a frame size of 100 samples with a step size of 2 samples, using Support Vector Machine (SVM) classifier.
\end{abstract}

Keywords: Eigenfunctions of the Schrödinger operator, Epilepsy, Machine Learning, spikes detection

\section{INTRODUCTION}

The presence of peaks in biomedical signals often reflects different chemical and biological activities in the human body. An example of such signals is the Magnetoencephalography (MEG) signals. The MEG is a functional neuroimaging modality that measures the magnetic activity of the brain. It uses an array of highly sensitive sensors or magnetometers called superconducting quantum interference devices (SQUIDs). The MEG signal is less distorted by the intervening tissues between the neural source and the SQUIDs comparing to electroencephalogram (EEG) signal for example [1]. MEG signals are very useful for the detection and the treatment of epileptogenic zone in the brain [2]. Epileptic patients often suffer from unpredictable unprovoked seizures that may affect their activity. For instance, epileptic patients stare blankly for a few seconds during a seizure, while others have uncontrollable jerking movements of the arms and legs. MEG signals help in localizing the region of the brain which produces the abnormal electrical activities that causes the neurological disorder. If the localized region is not responsible for a vital function in the brain such as speech, then this region is usually extracted surgically. In addition, the MEG is used for the assessment of the patient state after a surgery.

Due to the recent introduction of the MEG modality in clinical practice, the interictal epileptic spike detection using MEG signals is an emerging research field. Different methods were proposed for epileptic spikes detection using EEG/MEG signals [3]. However, to the best of the authors knowledge, few methods exists for spike detection using the MEG signal [4], [5], [6]. For example, in [4], an independent component analysis (ICA) method has been proposed with a multi-channel MEG spikes localization method, which decomposes spike-like and background components into separate spatial topographies and associated time series. The detection is performed using a thresholding technique. Another method is the common spatial patterns (CSP) and linear discriminant analysis (LDA) method (CSP-LDA) [5]. The latter is similar to the method in [4] except in the classification stage where the LDA classifier is used for the detection. Moreover, an Amplitude Thresholding and Dynamic Time Warping (AT-DTW) approach has been proposed in [6]. This method uses amplitude thresholding to localize abnormal activities, $\mathrm{F}$ to specify the region in the brain where the abnormalities happen and to select the affected channels. For the spike detection, the method employs dynamic time warping. The previously cited works reported a maximum sensitivity and specificity of $92.4 \%$ and $95.8 \%$, respectively. Most of these methods study the MEG signal in its time domain, and some of them do not take advantage of recent advances in machine learning classifiers due to the complexity of the MEG signal. Therefore, Pertinent characterization of MEG signals, which allows the definition of discriminative features, is important for an effective use of classifiers for spike detection. It is also important that the introduced feature vector is of reduced dimension to improve the effectiveness of the classification.

In this paper, we propose a novel feature generation and dimensionality reduction method for multi-channel MEG signal, which improves patient-independent spike detection. 


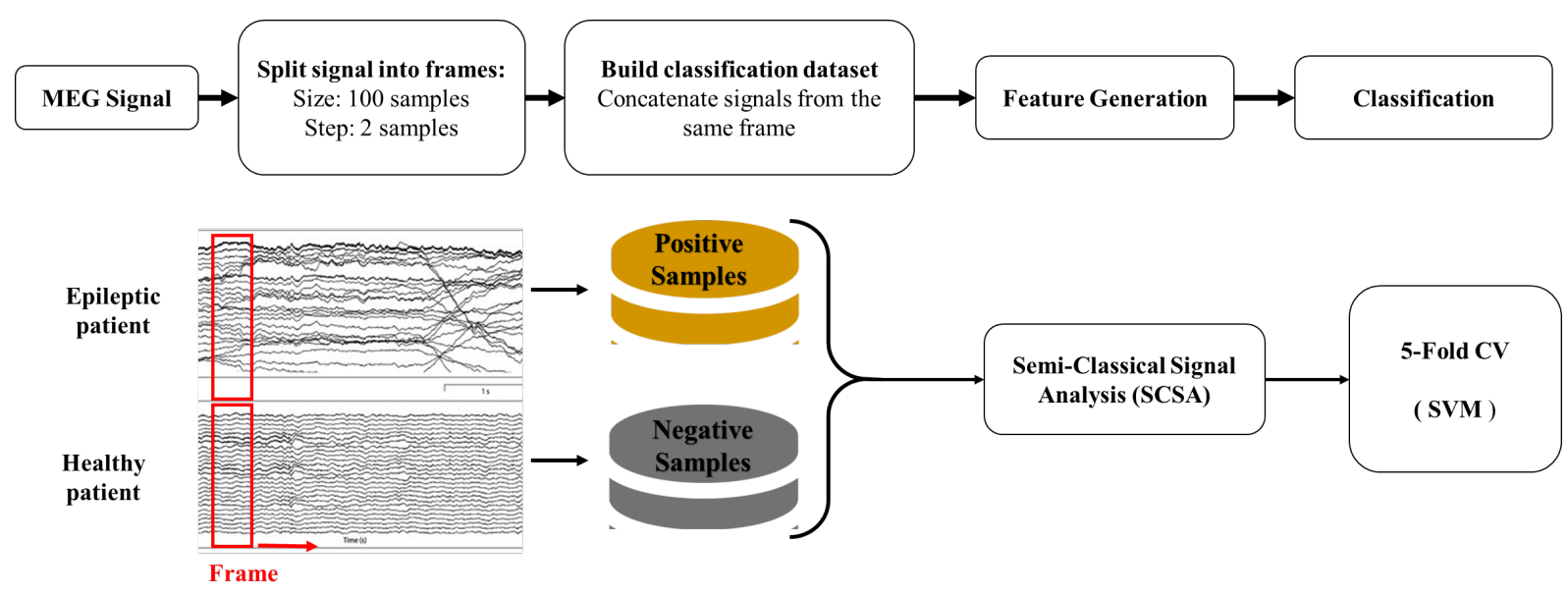

Fig. 1. Classification framework: MEG records pre-processing, feature generation and classification models.

This method is based on the discrete spectrum of the Schrödinger operator. This study shows a great potential in reducing the dimensionality of the feature vector and improving the accuracy of the epileptic spike detection.

The paper is organized as follows: we first describe MEG dataset and the proposed features extraction method based on the Schrödinger operator spectrum in Section 2. The obtained results using eight healthy and eight epileptic patients are presented and discussed in Section 4. Finally, Section 5 gives our concluding remarks about the proposed method and the future work.

\section{METHODS \& MATERIALS}

\section{A. MEG data acquisition and analysis}

MEG data were recorded in a shielded room at National Neural Institute (NNI) at King Fahad Medical City (KFMC) in Riyadh (Saudi Arabia) with an Elekta Neuromag system. Elekta Neuromag head system (helmet) contains 102 magnetometer and 204 gradiometer sensors. These sensors are further categorized according to the different brain regions. Each element of the Elekta Neuromag system is comprised of three sensors, one magnetometer, and two gradiometers. Magnetic brain activity was recorded at a sampling frequency of $1 \mathrm{kHz}$. MEG data was filtered by tSSS (Spatiotemporal signal space separation) method [7]. The data were then off-line band-pass filtered $1-50 \mathrm{~Hz}$ for visual inspection. A total of 18 MEG data segments, each of 15 minutes duration and 26 channels, were taken from eight epileptic patients and eight healthy patients. These segments are analyzed by expert neurologists from NNI. The neurologists marked the MEG spikes locations, in different brain regions, by visual inspection. The total number of spikes in these recordings is 166 . Written informed consent was signed by each participant or responsible adult before participation. The study was conducted in accordance with the approval of the Institutional Review Board at KFMC (IRB log number: 15086, 2015).

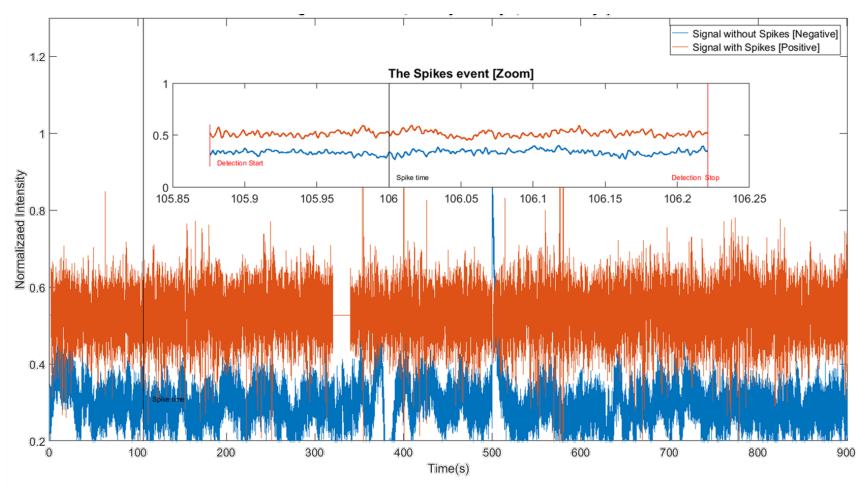

Fig. 2. Example of spikes in one of the MEG channels.

\section{B. $M E G$ signal framing}

The multi-channel MEG signals are segmented into frames using sliding frames, where a sliding segment of size 100 sample points with step $=2$ samples as shown in Figures 1 and 2. The frames are used to extract the signals corresponding to the different classes defined as follows:

1) Positive class: includes the MEG spike candidate extracted from the annotated spikes regions from the 24 electrodes of the 8 epileptic patients.

2) Negative class: includes the same number of frames as the positive class extracted from uniformly distributed locations from 24 electrodes of the 8 healthy patients.

\section{Feature generation and dimensionality reduction}

1) Semi-Classical Signal Analysis method: The SemiClassical Signal Analysis (SCSA) method uses signaldependent functions given by the squared eigenfunctions of the Schrödinger operator to decompose the signal as introduced in [8] and [9]. The potential of the Schrödinger operator $H(y)$, in this case, is given by the positive function $y(t)$ representing the signal.

$$
H(y)=-h^{2} \frac{d^{2}}{d t^{2}}-y(t)
$$


with,

$$
H(y) \psi(t)=\lambda \psi(t)
$$

The Semi-Classical Signal Analysis shows that a real positive input signal $y(t)$ can be approximated by $y_{h}(t)$ given in the following form:

$$
y_{h}(t)=4 h \sum_{n=1}^{N_{h}} \sqrt{-\lambda_{n h}} \psi_{n h}^{2}(t),
$$

where $h \in \mathbb{R}_{+}^{*}, \lambda_{n h}$ and $\psi_{n h}(t)$, for $n=1, \cdots, N_{h}$, refer to the negative eigenvalues of the semi-classical Schrödinger operator $H(y)$, with $\lambda_{1 h}<\cdots<\lambda_{N_{h h}}<0$, and their associated $L^{2}$-normalized eigenfunctions, respectively.

Equation (3) provides an exact reconstruction of the signal when $h$ converges to zero. This was the reason for calling this method semi-classical signal analysis or SCSA where $h$ is called semi-classical parameter. Moreover, when $h$ decreases, the number of eigenvalues increases and the reconstruction improves, as explained in [8]. The algorithm of the SCSA method for signal reconstruction has been reported in [8] and is given by:

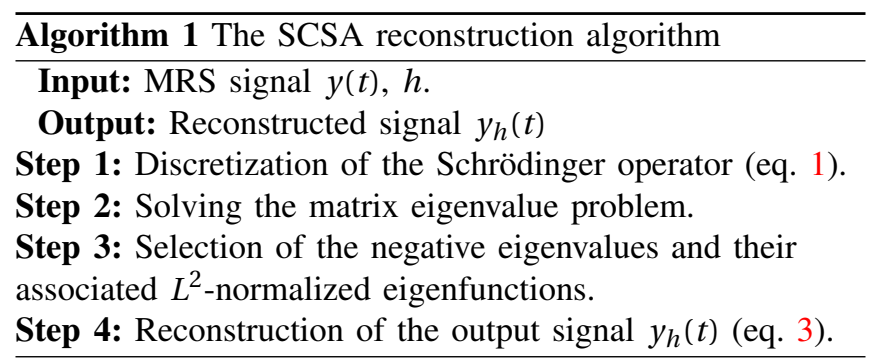

2) SCSA for feature dimensionality reduction: The SCSA method has been used for reconstruction and denoising of some bio-medical signals such as the Magnetic Resonance Spectroscopy (MRS) spectra and the Arterial Blood Pressure (ABP) [10], [11]. In general and thanks to the localized and shape-dependent structure of the squared eigenfunctions of the Schrödinger operator, the SCSA introduces an effective analysis tool for pulse shaped signals (signals with peaks) [11]. In this paper, we investigate the usefulness of the SCSA in defining new characteristics of MEG signals. In particular, we study the pertinence of reducing the order of the feature vector which can be extracted from the discrete spectrum of the Schrödinger operator after accurately reconstructing the signal (Figure 3). As discussed in the previous subsection, it is important to choose an appropriate value of the parameter $h$ to ensure an accurate reconstruction of the signal and thus a reliable projection to the new spectral dataset given by the negative eigenvalues and the associated eigenfunctions. In particular, we will pay attention to the appropriate choice of the dimension of our negative spectrum, which has to be the same for all projected signals. Hence, we introduce $N_{h}^{\star}$ as the lower feature size as follows:

$$
N_{h}^{\star}=\min \left(N_{h 1}, N_{h 2}, \ldots N_{h M}\right)
$$

were $N_{h i}, i=1, \cdots, M$ is the number of negative eigenvalues of the $i^{t h}$ decomposed frame using an appropriate value $h$. Therefore, the generated features are the first $N_{h}^{\star}$ negative eigenvalues for each dataset's frame.

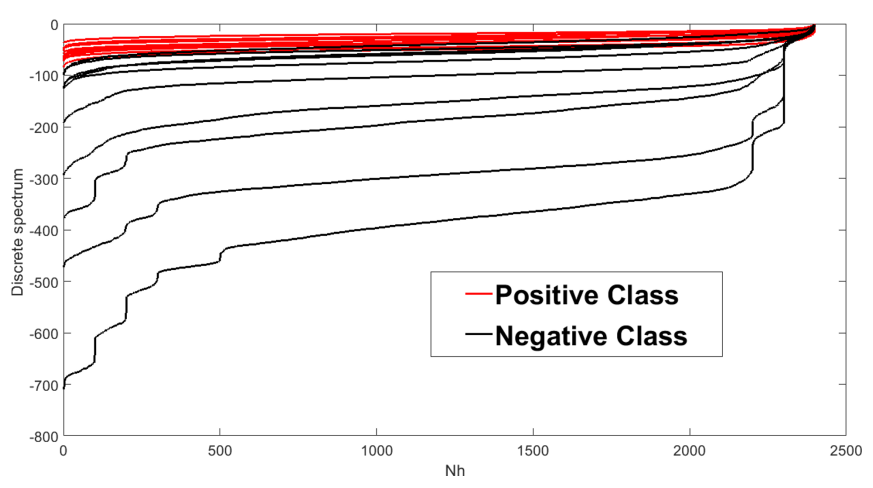

Fig. 3. Example of the discrete spectrum of the Schrödinger operator for positive frames and negative frames using $h=0.5$

\section{Epileptic Spike detection}

1) Classification models: For each of the two datasets described in section II-A, the SCSA-based features were used to feed the Support Vector Machine (SVM) predictive model. The SVM model was developed in 5-fold cross-validation (CV) process with subjects: 1734 spiky frames and 1734 healthy frames from different MEG test sessions of eight healthy and eight epileptic patients.

2) Evaluation: The performance of SVM model has been measured using the average accuracy, the sensitivity, the specificity and other metrics defined as follows:

$$
\begin{gathered}
\text { Accuracy }=\frac{1}{5} \sum_{n=1}^{5} \frac{T P_{n}+T N_{n}}{T P_{n}+F P_{n}+T N_{n}+F N_{n}} \times 100 \\
\text { Sensitivity }=\frac{1}{5} \sum_{n=1}^{5} \frac{T P_{n}}{T P_{n}+F N_{n}} \times 100 \\
\text { Specificity }=\frac{1}{5} \sum_{n=1}^{5} \frac{F P_{n}}{F P_{n}+T N_{n}} \times 100 \\
\text { Precision }=\frac{1}{5} \sum_{n=1}^{5} \frac{T P_{n}}{T P_{n}+F P_{n}} \times 100
\end{gathered}
$$

$$
\begin{gathered}
\text { Gmean }=\sqrt{\text { Sensitivity } \times(1-\text { Specificity })} \\
F_{1}-\text { Score }=\frac{2 \times \text { Precision } \times \text { Sensitivity }}{\text { Precision }+ \text { Sensitivity }}
\end{gathered}
$$

where $T P_{n}, F P_{n}, T N_{n}, F N_{n}$ are the True Positive, False Positive, True Negative and False Negative respectively in the $n^{\text {th }}$ fold.

\section{RESULTS \& DISCUSSION}

The SCSA-based features depend on the choice of $h$. Table I shows that with the same average number of negative eigenvalues $N_{h}^{\star}$, lower values of $h$ improves the classification performance. This is an expected result since lower values 
TABLE I

THE EFFECT OF $h$ VALUE ON THE CLASSIFICATION PERFORMANCE.

\begin{tabular}{|c|c|c|c|c|c|c|c|}
\hline $\begin{array}{c}\mathbf{h} \\
\text { value }\end{array}$ & $N_{h}^{\star}$ & Accuracy & Sensitivity & Specificity & Precision & Gmean & F1-Score \\
\hline 0.001 & 1 & 90.88 & 92.52 & 89.23 & 89.61 & 90.86 & 91.03 \\
\hline 0.01 & 1 & 90.68 & 92.52 & 88.84 & 89.27 & 90.66 & 90.85 \\
\hline 0.1 & 1 & $\mathbf{9 0 . 8 8}$ & $\mathbf{9 2 . 5 2}$ & $\mathbf{8 9 . 2 3}$ & $\mathbf{8 9 . 6 0}$ & $\mathbf{9 0 . 8 6}$ & $\mathbf{9 1 . 0 3}$ \\
\hline 0.3 & 1 & 90.72 & 92.52 & 88.91 & 89.31 & 90.69 & 90.88 \\
\hline 0.5 & 1 & 90.78 & 92.52 & 89.04 & 89.42 & 90.76 & 90.93 \\
\hline 1 & 1 & 90.68 & 92.46 & 88.91 & 89.30 & 90.66 & 90.85 \\
\hline 10 & 2 & 89.39 & 92.78 & 86.01 & 86.95 & 89.31 & 89.74 \\
\hline 50 & 2 & 89.36 & 91.75 & 86.98 & 87.60 & 89.32 & 89.62 \\
\hline
\end{tabular}

TABLE II

COMPARISON OF THE PROPOSED METHODS TO EXISTING METHODS RECENTLY REPORTED IN [6]

\begin{tabular}{|c|c|c|c|c|c|c|}
\hline Method & $\begin{array}{c}\# \\
\text { Patients }\end{array}$ & Year & Feature & Accuracy & Sensitivity & Specificity \\
\hline ICA [4] & 4 & 2004 & $\begin{array}{c}\text { Independent Components } \\
\text { Analysis }\end{array}$ & - & 86.91 & 81.19 \\
\hline CSP-LDA [5] & 20 & 2016 & $\begin{array}{c}\text { Common Spatial Patterns and } \\
\text { Linear Discriminant Analysis }\end{array}$ & - & 86.14 & 90.38 \\
\hline AT-DTW [6] & 30 & 2017 & $\begin{array}{c}\text { Amplitude Thresholding and } \\
\text { Dynamic Time Warping }\end{array}$ & - & 92.45 & $\mathbf{9 5 . 8 1}$ \\
\hline SCSA-SVM & 16 & 2019 & $\begin{array}{c}\text { Semi-Classical Signal Analysis } \\
\text { and Support Vector Machine }\end{array}$ & 90.88 & $\mathbf{9 2 . 5 2}$ & 89.23 \\
\hline
\end{tabular}

of $h$ improves the accuracy of the reconstruction as shown in [8]. However, the value of $h$ cannot be very small due to the limited number of points, which limits the number of negative eigenvalues that can be computed numerically.

The proposed method is compared to the existing spikes detection approaches using MEG signal reported in this work [6]. Table II shows that the SCSA-based features improve the detection sensitivity and gets higher specificity compared to two of the existing methods. Moreover, the proposed method reduces the feature size to one. This fact is shown in Figure 3 , which shows that the spectrum of Schrödinger operator separates the positive class from the negative class as we use the first negative eigenvalues as features which is the most dominant and representative ones.

\section{CONCLUSiON}

We developed a new feature generation and dimensionality reduction method for epileptic spikes detection in MEG signals. This method projects the input signal into the discrete spectrum of the Schrödinger operator. The proposed method obtains the highest Sensitivity up to $92.52 \%$ with a specificity of $89.10 \%$ for a dataset consisting of 8 healthy and 8 epileptic patients. As a future work, we aim to take advantage from the Schrödinger operator eigenfunctions in order to improve the detection performance.

\section{ACKNOWLEDGEMENT}

Research reported in this publication was supported by King Abdullah University of Science and Technology (KAUST)Base Research Fund (BAS/1/1627-01-01), in collaboration with King Abdulaziz City for Science and Technology (KACST) and King Saud University (KSU).

\section{REFERENCES}

[1] F. L. da Silva, "EEG and MEG : Relevance to neuroscience," Neuron, vol. 80 , no. 5, pp. $1112-1128,2013$.

[2] D. J. Englot, S. S. Nagarajan, B. S. Imber, K. P. Raygor, S. M. Honma, D. Mizuiri, M. Mantle, R. C. Knowlton, H. E. Kirsch, and E. F. Chang, "Epileptogenic zone localization using magnetoencephalography predicts seizure freedom in epilepsy surgery," Epilepsia, vol. 56, no. 6, pp. 949-958, jun 2015.

[3] F. E. A. El-Samie, T. N. Alotaiby, M. I. Khalid, S. A. Alshebeili, and S. A. Aldosari, "A review of EEG and MEG epileptic spike detection algorithms," IEEE Access, vol. 6, pp. 60 673-60 688, 2018.

[4] A. Ossadtchi, S. Baillet, J. C. Mosher, D. Thyerlei, W. Sutherling, and R. M. Leahy, "Automated interictal spike detection and source localization in magnetoencephalography using independent components analysis and spatio-temporal clustering," Clinical Neurophysiology, vol. 115, no. 3, pp. 508-522, 2004.

[5] M. I. Khalid, T. Alotaiby, S. A. Aldosari, S. A. Alshebeili, M. H. Al-Hameed, F. S. Y. Almohammed, and T. S. Alotaibi, "Epileptic MEG Spikes Detection Using Common Spatial Patterns and Linear Discriminant Analysis," IEEE Access, vol. 4, pp. 4629-4634, 2016.

[6] M. I. Khalid, T. N. Alotaiby, S. A. Aldosari, S. A. Alshebeili, M. H. Alhameed, and V. Poghosyan, "Epileptic MEG Spikes Detection Using Amplitude Thresholding and Dynamic Time Warping," IEEE Access, vol. 5, pp. 11658-11667, 2017.

[7] S. Taulu and J. Simola, "Spatiotemporal signal space separation method for rejecting nearby interference in MEG measurements," Physics in Medicine and Biology, vol. 51, no. 7, pp. 1759-1768, 2006.

[8] T.-M. Laleg-Kirati, E. Crépeau, and M. Sorine, "Semi-classical signal analysis," Mathematics of Control, Signals, and Systems, vol. 25, no. 1, pp. 37-61, 2013.

[9] B. Helffer and T.-M. Laleg-Kirati, "On semi-classical questions related to signal analysis," Asymptotic Analysis.

[10] T.-M. Laleg, E. Crépeau, Y. Papelier, and M. Sorine, "Arterial blood pressure analysis based on scattering transform i," in Engineering in Medicine and Biology Society, 2007. EMBS 2007. 29th Annual International Conference of the IEEE. IEEE, 2007, pp. 5326-5329.

[11] T. Laleg-Kirati, J. Zhang, E. Achten, and H. Serrai, "Spectral data denoising using semi-classical signal analysis: application to localized MRS," NMR in Biomedicine, vol. 29, no. 10, pp. 1477-1485, 2016. 